\title{
A fourth dimension to the stromal scaffold
}

DOI:

10.1038/nri2207
Fibroblastic reticular cells (FRCs) provide a three-dimensional scaffold on which immune cells travel as they negotiate their way through secondary lymphoid organs in search of cognate antigen. Such FRC stromal networks are therefore crucial for proper lymphoid architecture and function. Now, FRCs have been assigned two new roles: producers of the key T-cell survival factor interleukin-7 (IL-7) and targets of persistent viral infection.

IL-7 is known to be essential for naive $\mathrm{T}$-cell survival and previous studies hinted that this signal may be provided in the secondary lymphoid organs. Consistent with this, when Link et al. blocked the access of lymphocytes to lymph nodes, they saw a deleterious effect on T-cell survival, which was similar to the effect of blocking IL-7. To pinpoint the main source of the IL-7, the authors measured $I l 7$ mRNA expression in various

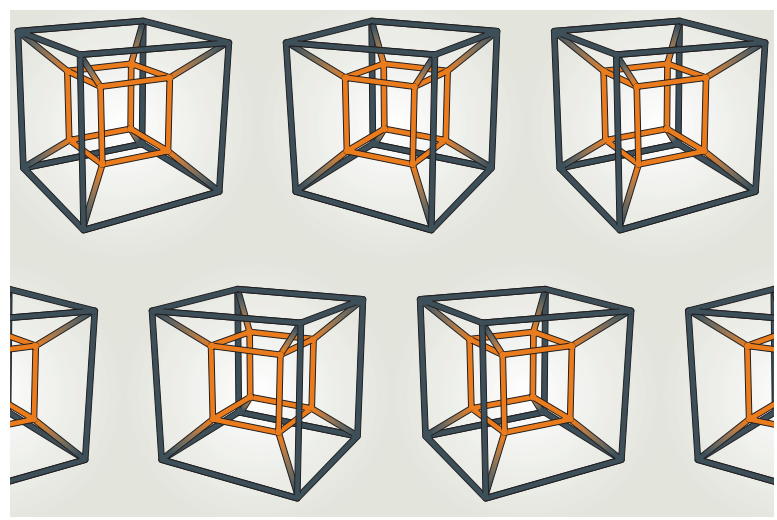

lymph-node fractions, including dendritic-cell fractions and three stromal-cell fractions. FRCs in the T-cell zone (characterized as gp $\left.38^{+} \mathrm{CD} 31^{-} \mathrm{CD} 35^{-} \mathrm{CD} 45^{-}\right)$were revealed as having more $I l 7 \mathrm{mRNA}$ expression than any other lymphnode cell type. These cells also contained high levels of transcripts encoding CC-chemokine ligand 19 (CCL19) and CCL21, confirming the role of these cells in supporting T-cell migration in secondary lymphoid organs.

In vitro, an adherent layer of lymph-node stromal cells enriched for T-cell-zone FRCs were able to support naive T-cell survival, and the survival effect was halved in the presence of IL-7-specific blocking antibody. Blocking of both CCL19 and IL-7 further reduced the survival effect of stromal cells in vitro, suggesting that these two factors have an additive effect on T-cell survival. Analysis of

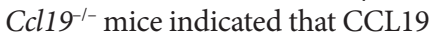
in fact has a non-redundant function in naive T-cell homeostasis but is less important than IL-7.

In the study by Mueller et al., FRCs were shown to be the preferred cell type targeted by the strain of lymphocytic choriomeningitis virus (LCMV) that establishes chronic infection in mice, LCMV clone 13, and not by the acute strain, LCMV Armstrong. Chronic LCMV infection is known to be associated with persisting virus, disrupted lymphoid architecture and generalized immuno suppression, so the authors asked whether the infection of FRCs might contribute to these features. First, they showed that the integrity of the conduit network (which transports small molecules and antigens to T-cell zones) formed by FRCs was disrupted in mice infected with LCMV clone 13. This was not the result of direct FRC cytotoxicity (as LCMV is non-cytolytic) but was caused by the responding $\mathrm{CD}^{+} \mathrm{T}$ cells. Second, they showed that, in mice infected with LCMV clone 13, FRCs expressed high levels of programmed cell death ligand 1 (PDL1), which is thought to inhibit T-cell function by binding PD1. Importantly, PDL1 expression by FRCs seemed to protect the FRCs from immunopathology, because in vivo blockade of the PD1-PDL1 pathway caused severe immunopathology and disruption of the FRC network in the spleens of mice infected with LCMV clone 13.

So, besides contributing to lymphoid tissue microarchitecture, FRCs seem to be active players in immune homeostasis.

Lucy Bird

ORIGINAL RESEARCH PAPERS Link, A. et al. Fibroblastic reticular cells in lymph nodes regulate the homeostasis of naive T cells. Nature Immunol. 23 September 2007 (doi:10.1038/ ni1513) | Mueller, S. N. et al. Viral targeting of fibroblastic reticular cells contributes to immunosuppression and persistence during chronic infection. Proc. Natl Acad. Sci. USA 104, 15430-15435 (2007) 